\title{
Confiabilidad y validez de un cuestionario de desarrollo infantil en encuestas nacionales
}

\author{
Reliability and validity of a questionnaire of child development for national surveys
}

Diana Marina Camargo Lemos ${ }^{a}$, María Solange Patiño Segura ${ }^{a}$, Yuri Liseth Sánchez Martínez ${ }^{\mathrm{a}}$

aEscuela de Fisioterapia, Universidad Industrial de Santander, Colombia

Recibido: 24 de septiembre de 2018; Aceptado: 24 de octubre de 2019

¿Qué se sabe del tema que trata este estudio?

Latinoamérica tiene poca información publicada sobre la situación del desarrollo infantil en su población, posiblemente por la falta de un instrumento de tamizaje válido y confiable, que permita establecer alteraciones del desarrollo y plantear políticas públicas para su atención oportuna.
¿Qué aporta este estudio a lo ya conocido?

Evidencia sobre la confiabilidad y validez de constructo convergente del Cuestionario de Tamizaje de Desarrollo Infantil para Encuestas en Hogares (DIEH), como un instrumento potencial para ser usado en encuestas nacionales de manera rápida y con bajo costo.

\section{Resumen}

El tamizaje del desarrollo infantil en encuestas nacionales es un insumo para la formulación de políticas públicas que contribuyan a su diagnóstico e intervención precoz, dirigidos a favorecer un desarrollo integral y desempeño escolar exitoso hasta la adolescencia, no obstante, pocos países en Latinoamérica cuentan con esta información. Objetivo: Evaluar la confiabilidad y validez convergente de una adaptación cultural del Cuestionario de Tamizaje de Desarrollo Infantil para Encuestas en Hogares (DIEH) y describir la prevalencia de alteraciones en el desarrollo en niños de 2 meses a 5 años en Bucaramanga-Colombia. Sujetos y Método: Se realizó una evaluación de pruebas diagnósticas. Participaron 224 niños de dos meses hasta los cinco años y sus padres o cuidadores procedentes de Bucaramanga - Colombia, entre julio y diciembre de 2016. La Escala Abreviada del Desarrollo (EAD) fue aplicada por fisioterapeutas entrenados y el DIEH, fue respondido por los padres o cuidadores. A partir del alpha de Cronbach, el Coeficiente de Correlación Intraclase (CCI) y los límites de acuerdo Bland y Altman se estableció la confiabilidad del DIEH; con el Coeficiente de Correlación de Spearman la validez convergente. Resultados: A partir del DIEH las prevalencias de rezagos y retrasos fueron $38.8 \%$ y $11.2 \%$ respectivamente; la consistencia interna osciló entre 0.23 y 0.76 . La reproducibilidad registró CCI entre 0.60 y 0.92 ; la validez convergente fue casi perfecta ( $\rho$ : 0.96). Conclusiones: La adaptación cultural del DIEH mostró propiedades psicométricas aceptables, que podrían complementarse con estudios adicionales para recomendar su uso en encuestas nacionales en Latinoamérica.
Palabras clave: Desarrollo infantil; tamizaje; niños; encuestas y cuestionarios; reproducibilidad de los resultados 


\begin{abstract}
The child development screening in national surveys supports the formulation of public policies that contribute to its diagnosis and early intervention, aimed at promoting comprehensive development and successful school performance until adolescence, however, few countries in Latin America have this information. Objective: To assess the reliability and convergent validity of a cultural adaptation of the Screening Questionnaire of Child Development for Household Surveys (DIEH) and to describe the prevalence of developmental alterations in children aged between 2 months and 5 years in Bucaramanga-Colombia. Subjects and Method: Between July and December 2016, an evaluation of diagnostic tests was carried out in which participated 224 children aged between two months and five years and their parents or caregivers from Bucaramanga, Colombia. The Abbreviated Developmental Scale was applied by trained physiotherapists and the DIEH was answered by the parents or caregivers. The reliability of the DIEH was established using the Cronbach's alpha, the Intraclass Correlation Coefficient (ICC) and the Bland and Altman limits of agreement, and the convergent validity was established through the Spearman Correlation Coefficient. Results: Based on the DIEH, the prevalence of lags and delays was $38.8 \%$ and $11.2 \%$ respectively; internal consistency ranged from 0.23 to 0.76 ; reproducibility showed an ICC between 0.60 and 0.92 ; and the convergent validity was almost perfect ( $\rho: 0.96)$. Conclusion: The cultural adaptation of the DIEH showed acceptable psychometric properties, which could be complemented with additional studies to recommend its use in national surveys in Latin America.
\end{abstract}

Keywords:

Child development; screening; children; surveys and questionnaires; results reproducibility

\section{Introducción}

El proceso de desarrollo se caracteriza por su dinámica de cambio constante, diferenciado por etapas de complejidad creciente y consecutiva ${ }^{1,2}$, fundamentales para el desempeño en la vida adulta ${ }^{3}$. Incluye diversos dominios interdependientes: lenguaje-cognitivo, sensorial-motor y social-emocional, predictores de logros académicos, productivos y de funcionamiento social en el transcurso de la vida ${ }^{4}$.

La interacción entre la carga genética del individuo, su condición biológica y el medio ambiente familiar y social interactúan en el proceso de desarrollo infantil ${ }^{2}$, que se caracteriza por su rápida evolución particularmente en los primeros años de vida ${ }^{1}$, etapa primordial en la cual, situaciones de desventaja socio económica pueden generar un impacto importante sobre su proceso de evolución ${ }^{5}$, en especial en países con bajos y medianos ingresos, donde se estima que $43 \%$ de los niños están en condición de riesgo debido a la pobreza extrema ${ }^{6}$.

Los trastornos del desarrollo son cuadros crónicos y de inicio precoz que provocan un impacto negativo y significativo en el progreso del desarrollo de los niños y se evidencian cuando no alcanzan los logros esperados para la edad ${ }^{7,8}$. Las prevalencias en América Latina, según encuestas realizadas a nivel local, en San IsidroArgentina ${ }^{9}$; estatal, en Querétaro-México ${ }^{10}$ y nacional en Chile ${ }^{11}$ oscilan entre $20 \%$ y $36 \%$; Colombia no cuenta con esta información.

Las alteraciones en el desarrollo pueden clasificarse en dos categorías: retraso, entendido como la adquisi- ción de los hitos motores de una forma más lenta de la esperada para la edad ${ }^{12}$ y trastornos, relacionados con la adquisición de patrones anormales durante el desarro$1 \mathrm{lo}^{13}$; su detección precoz permite intervenir oportunamente para mejorar el pronóstico de los niños ${ }^{1,12,14}$.

Los test de tamizaje del desarrollo infantil están dirigidos a identificar el riesgo de presentar algún tipo de retraso, permitiendo priorizar el acceso a servicios médicos, con el fin de promover la atención temprana de los niños ${ }^{1,15}$ mediante valoraciones transitorias, que deben validarse con pruebas de diagnóstico para confirmar o rechazar los hallazgos de la exploración inicial ${ }^{8}$. No obstante, varios obstáculos como la falta de tiempo durante la consulta de atención primaria y la insuficiencia de personal entrenado para la aplicación de pruebas hacen difícil su aplicación ${ }^{14,16}$.

En Colombia la evaluación y seguimiento del desarrollo de los niños se realiza mediante la Escala Abreviada del Desarrollo (EAD), que evalúa cuatro áreas específicas ${ }^{17}$, requiere de personal entrenado, así como un tiempo promedio de 20 minutos para su aplicación, lo que posiblemente ha dificultado su uso en los programas de crecimiento y desarrollo. En relación con sus propiedades psicométricas y con base en la literatura consultada, solo un estudio evaluó la validez convergente entre la EAD y el Neurosensory Motor Development Assessment, encontrando una correlación moderada $(r=0,51)$ entre estos dos instrumentos ${ }^{18}$.

Diversos cuestionarios de tamizaje, algunos dirigidos a los padres y otros al médico de atención primaria o pediatra, permiten detectar en forma rápida, sencilla y a bajo costo, posibles alteraciones en el desarrollo es- 
perado para la edad ${ }^{12,19}$, cualidades indispensables para ser usados en encuestas nacionales, que requieren, además, un mínimo entrenamiento para los encuestadores. No obstante, es necesario que cuenten con propiedades psicométricas aceptables.

La Encuesta Nacional de Calidad de Vida y Salud (ENCAVI) de 2006 en Chile ${ }^{11}$, utilizó el Cuestionario de Tamizaje de Desarrollo Infantil para Encuestas en Hogares (DIEH) diligenciado por el cuidador principal, con el fin de evaluar el desarrollo de los niños entre 2 meses a 5 años y 11 meses de edad. Este cuestionario, fue creado ante la necesidad de contar con un instrumento para valorar el desarrollo en la primera infancia a muy bajo costo, sin los requerimientos de pruebas de diagnóstico más específicas y con el propósito de registrar una cifra estimada de la situación del desarrollo de los niños. Cuenta con una sensibilidad de $88 \%$ y una razón de probabilidad positiva de 1,96 comparada con el inventario de desarrollo de Battelle- $2^{20}$.

Lo autores de la DIEH han propuesto su uso en otras regiones, previa adaptación cultural, lo que permitiría conocer la situación global del desarrollo de los niños en encuestas poblacionales en el ámbito nacional $^{20}$, por esta razón, el objetivo de este estudio fue evaluar la consistencia interna, reproducibilidad testretest, el nivel de acuerdo y la validez de constructo de tipo convergente, de una adaptación cultural para Colombia de la DIEH y, adicionalmente, describir la prevalencia de retraso en el desarrollo en niños de 2 meses a 5 años en Bucaramanga-Colombia.

\section{Sujetos y Método}

Se realizó una evaluación de pruebas diagnósticas con un muestreo de corte transversal entre julio y diciembre de 2016.

\section{Población de estudio}

La población estuvo conformada por niños entre 2 y 60 meses de edad y sus padres, seleccionados por conveniencia en tres Centros de Desarrollo Infantil, adscritos al Instituto Colombiano de Bienestar Familiar y otras instituciones de preescolar del municipio de Bucaramanga.

Los representantes legales de los tres Centro de Desarrollo Infantil, así como los padres o acudientes de cada menor, firmaron el consentimiento informado para autorizar la participación de los niños. El estudio contó con el aval del Comité de Ética de la Universidad Industrial de Santander.

\section{Variables de estudio}

Se recolectó información sociodemográfica de los niños y se indagó por el régimen de seguridad social al que pertenecían, clasificado en dos categorías: contri- butivo, se refiere a la vinculación mediante el pago de una cotización o aporte económico financiado directamente por el afiliado ${ }^{21}$ y subsidiado, corresponde al mecanismo por el cual la población más pobre del país, sin capacidad de pago, tiene acceso a los servicios de salud mediante un subsidio que ofrece el Estado ${ }^{22}$. Adicionalmente, se preguntó si contaban con los carnets de vacunación y del programa de crecimiento y desarrollo, el tiempo de vinculación y la periodicidad de las valoraciones. También, se indagó por el peso al nacer y el nacimiento a término mediante información de la madre o cuidador. Además, se registró la edad de la madre en el momento de nacimiento del niño, el número de hijos nacidos vivos y el estrato socioeconómico entre 1 y 6 , entendiendo como 1 el más bajo y 6 el más alto.

\section{Desarrollo infantil}

La Escala Abreviada del Desarrollo (EAD) fue seleccionada como prueba de referencia a pesar de no ser reconocida como "gold estándar", ya que es el instrumento definido para Colombia según la Norma Técnica para la detección temprana de las alteraciones del crecimiento y desarrollo en menores de 10 años (Resolución 412 del Ministerio de Salud de Colombia, año $2000)^{23,24}$.

La EAD evalúa el desarrollo infantil en niños de 1 a 72 meses en 4 áreas específicas: motora gruesa, motora fina-adaptativa, audición-lenguaje y personal-social; cada una cuenta con 30 ítems, a los cuales se les asigna la calificación de [1] si el niño cumple con la actividad y [0] en caso contrario. La sumatoria de los puntajes de cada área y el total, clasifican al niño según la edad en cuatro categorías de desarrollo: alerta, medio, medio alto y alto; estableciendo en "alerta", una evaluación más exhaustiva para diagnosticar o descartar alteraciones o retrasos en el desarrollo ${ }^{17}$.

El Cuestionario de Tamizaje de Desarrollo Infantil para Encuestas en Hogares (DIEH) incluye de 4 a 9 ítems por grupo etario y cubre cuatro áreas del desarrollo: social, lenguaje, cognitiva y motora, que discrimina tres categorías de desarrollo: normal, rezago y retraso. La recolección de la información se realiza en forma secuencial, aplicando las preguntas una vez se ubique al niño en el módulo correspondiente a su edad, pero iniciando con las preguntas del tramo de edad anterior. De esta manera, cuando el padre o cuidador refiere que el niño no completa las actividades del tramo anterior a su grupo etario se califica como retraso y, cuando completa las actividades del tramo anterior a su grupo etario, pero no realiza todas las actividades para su grupo de edad, se califica como rezago; la realización de todas las actividades correspondientes a las de su grupo etario y del anterior, se clasifica en la categoría normal $^{3,20}$. Todas las respuestas se codifican de manera dicotómica (sí/no) ${ }^{3}$. 


\section{Procedimiento}

Debido a que el DIEH es un cuestionario de origen chileno, inicialmente se realizó una adaptación cultural con tres ajustes a los términos originales. En el tramo 3 (niños de 1 año a 23 meses) ítem 6, se cambió en el ejemplo el término "mostrame tu juguete favorito" por "muéstrame tu juguete favorito"; en el tramo 5 (niños de 3 años a 3 años 11 meses) ítem 2, se cambió en el ejemplo "vamos a las hamacas" por "vamos a jugar" y en el tramo 6 (niños de 4 años a 4 años 11 meses) ítem 9, se cambió en el ejemplo "va a buscar su campera" por "va a buscar su chaqueta".

Posteriormente, dos fisioterapeutas fueron entrenadas y se estandarizó el protocolo para aplicar los dos instrumentos. Se realizó una prueba piloto con tres niños por grupo etario, para un total de 24 , a quienes se les aplicó la EAD; todas las evaluaciones fueron registradas en video, con previo consentimiento del acudiente. Con base en este material audiovisual, tres días después de terminar la recolección de la información, se estableció la reproducibilidad entre evaluadores, obteniendo coeficientes Kappa $\geq 0,70$ para las 4 áreas evaluadas.

La recolección de la información se inició con una visita domiciliaria para la aplicación de la DIEH y se concertó una visita 7 días después, para la segunda evaluación; en el período intermedio, cada niño fue evaluado con la EAD durante la jornada escolar en los Centros de Desarrollo Infantil. Durante el proceso de evaluación cuatro niños entre los 13 y 24 meses se negaron a realizar las actividades de la EAD; adicionalmente, una niña de 49 a 60 meses realizó solo las actividades de motricidad gruesa.

\section{Análisis}

Se aplicó estadística descriptiva para presentar los hallazgos de las variables estudiadas. La consistencia interna se estableció con el Alpha de Cronbach, la reproducibilidad mediante el Coeficiente de Correlación Intraclase $\left(\mathrm{CCI}_{2,1}\right)$ y su interpretación siguió las recomendaciones de Landis y Koch ${ }^{25}$. Además, se determinó el nivel de acuerdo mediante el método de Bland y Altman ${ }^{26}$. La validez convergente del DIEH comparado con la EAD se estableció con el coeficiente de correlación de Spearman $(\rho)$ para los puntajes totales y por grupo etario. Cabe señalar que no fue posible comparar las categorías de: alerta, medio, medio alto y alto obtenidos a partir del EAD, con las categorías de: rezagos, retrasos y normal, derivados del DIEH. Esta dificultad obedeció principalmente al número de ítems asignado a cada dimensión por grupo de edad en los dos cuestionarios, que dejaban celdas sin participantes para aplicar el Kappa de Cohen. Todo el análisis se realizó con el software STATA $14.1(\alpha=0,05)$.

\section{Resultados}

\section{Descripción general}

Participaron 224 niños, 46,9\% mujeres, con una distribución homogénea por edad, a excepción del grupo de $49-60$ meses con $28,1 \%$ de los participantes. El $57,9 \%$ pertenecían al régimen contributivo y todos contaban con carnet de vacunación; en cuanto al Programa de Crecimiento y Desarrollo solo 96,9\% disponían de carnet, con una mediana de 30 meses en el programa y $50 \%$ registró dos visitas anuales. Las características sociodemográficas del cuidador y otros hallazgos adicionales se presentan en la tabla 1 .

\section{Prevalencias a partir de la DIEH}

Los resultados de la primera aplicación del DIEH

\begin{tabular}{|c|c|c|}
\hline \multirow{2}{*}{$\begin{array}{l}\text { Características sociodemográficas de los niños } \\
\text { Sexo femenino n (\%) }\end{array}$} & \multicolumn{2}{|c|}{$N=224$} \\
\hline & 105 & $(46.9)$ \\
\hline $\begin{array}{l}\text { Grupo etario } n(\%) \\
\begin{array}{l}2-6 \\
7-11 \\
12-23 \\
24-35 \\
36-47 \\
48-59\end{array}\end{array}$ & $\begin{array}{l}31 \\
36 \\
30 \\
35 \\
29 \\
63\end{array}$ & $\begin{array}{l}(13.8) \\
(16.1) \\
(13.4) \\
(15.6) \\
(13.0) \\
(28.1)\end{array}$ \\
\hline $\begin{array}{l}\text { Estrato socioeconómico n (\%) } \\
\qquad \begin{array}{l}1 \\
2 \\
3 \\
4 \\
5\end{array}\end{array}$ & $\begin{array}{c}70 \\
71 \\
56 \\
21 \\
6\end{array}$ & $\begin{array}{c}(31.2) \\
(31.7) \\
(25.0) \\
(9.4) \\
(2.7)\end{array}$ \\
\hline $\begin{array}{l}\text { Régimen de afiliación al sistema de seguridad social } r \\
\text { Contributivo } \\
\text { Subsidiado }\end{array}$ & $\begin{array}{l}\%) \\
129 \\
94\end{array}$ & $\begin{array}{l}(57.9) \\
(42.1)\end{array}$ \\
\hline Carné de vacunación n (\%) & 224 & $(100.0)$ \\
\hline Carné de crecimiento y desarrollo n (\%) & 217 & $(96.9)$ \\
\hline $\begin{array}{l}\text { Antecedentes clínicos de los niños n (\%) } \\
\text { Nacimiento a término } \\
\text { Complicaciones durante el embarazo } \\
\text { Características del cuidador }\end{array}$ & $\begin{array}{c}193 \\
62\end{array}$ & $\begin{array}{l}(86.2) \\
(27.7)\end{array}$ \\
\hline Sexo femenino $\mathrm{n}(\%)$ & 213 & $(95.1)$ \\
\hline Edad en años Media (DE) & 32 & $(10.9)$ \\
\hline Edad de la madre al momento del parto. Media (DE) & 26.1 & $(6.2)$ \\
\hline Escolaridad en años Media (DE) & 11.0 & $(3.0)$ \\
\hline $\begin{array}{l}\text { Estado civil n (\%) } \\
\text { Soltero/divorciado/viudo } \\
\text { Casado/unión libre }\end{array}$ & $\begin{array}{c}62 \\
162\end{array}$ & $\begin{array}{l}(27.7) \\
(72.3)\end{array}$ \\
\hline Número de hijos Mediana (Mínimo y Máximo) & 2 & $(1-7)$ \\
\hline $\begin{array}{l}\text { Número de hijos nacidos vivos Mediana (Mínimo y } \\
\text { Máximo) }\end{array}$ & 2 & $(1-7)$ \\
\hline
\end{tabular}




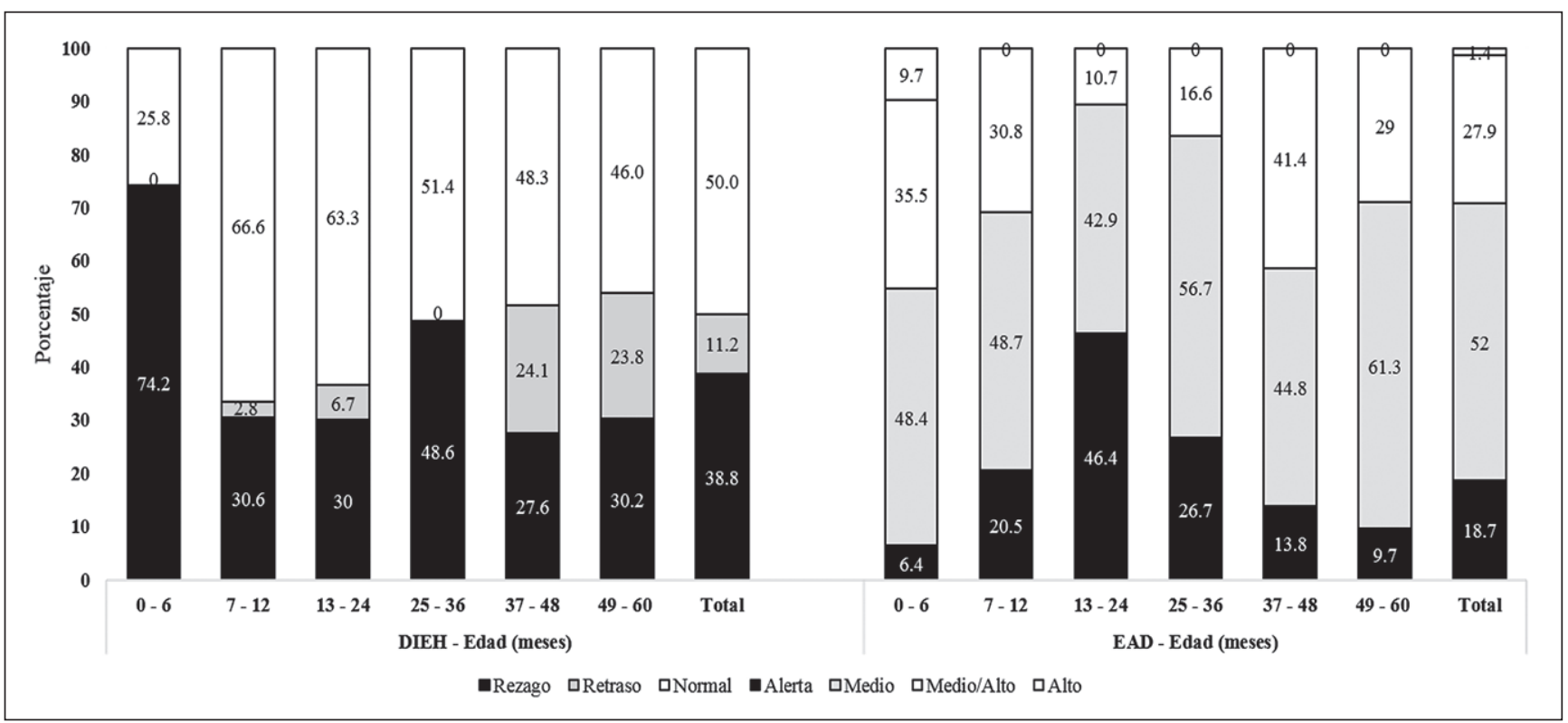

Figura 1. Resultados de la aplicación del Cuestionario de Tamizaje de Desarrollo Infantil para Encuestas en Hogares (DIEH) en la primera medición y de la Escala Abreviada del Desarrollo (EAD).

según el grupo etario se muestran en la figura 1, en la que se aprecia una mayor prevalencia de rezagos entre 0 y 6 meses $(74,2 \%)$, seguida por el grupo entre 25 y 36 meses $(48,6 \%)$; en general se registró una prevalencia de rezagos de $38,8 \%$ y de retrasos de $11,2 \%$.

\section{Prevalencias a partir de la EAD}

En la figura 1 se muestran los hallazgos de las cuatro categorías de la Escala Abreviada del Desarrollo por grupo etario, con las mayores prevalencias de alertas entre los 13 y 24 meses (46,4\%), seguido por el grupo entre 25 y 36 meses $(26,7 \%)$. En total, la prevalencia de alertas fue de $18,7 \%$ y en nivel medio $52,0 \%$.

\section{Confiabilidad del DIEH}

Los hallazgos de consistencia interna, reproducibilidad y acuerdo se muestran en el tabla 2 . Se encontró una consistencia interna entre baja $(0,23)$ para el grupo de 36 a 47 meses y buena $(0,76)$ para el grupo de 12 a 23 meses. La reproducibilidad mostró CCI en-

\begin{tabular}{|c|c|c|c|c|c|c|c|}
\hline \multirow{2}{*}{$\begin{array}{c}\text { Edad } \\
\text { (meses) }\end{array}$} & \multirow[t]{2}{*}{$\mathrm{n}$ niños } & \multirow[t]{2}{*}{$\mathrm{n}$ ítems } & \multirow[t]{2}{*}{$\alpha$-Cronbach } & \multicolumn{2}{|c|}{ Rezagos } & \multicolumn{2}{|c|}{ Retrasos } \\
\hline & & & & $\begin{array}{c}\mathrm{CCl} \\
\mathrm{IC} 95 \%\end{array}$ & $\begin{array}{c}\text { Promedio dif } \\
\left(L_{i}-L_{s}\right)\end{array}$ & $\begin{array}{c}\mathrm{CCl} \\
\mathrm{IC} 95 \%\end{array}$ & $\begin{array}{l}\text { Promedio dif } \\
\left(L_{i}-L_{s}\right)\end{array}$ \\
\hline $2-6$ & 31 & 5 & 0,47 & $\begin{array}{c}0,92 \\
0,87 ; 0,95\end{array}$ & $\begin{array}{c}0,0 \\
(-0,59 ; 0,59)\end{array}$ & --- & --- \\
\hline $7-11$ & 39 & 5 & 0,60 & $\begin{array}{c}0,80 \\
0,70 ; 0,87\end{array}$ & $\begin{array}{c}-0,08 \\
(-0,87 ; 0,72)\end{array}$ & $\begin{array}{c}1,0 \\
1,0 ; 1,0\end{array}$ & $\begin{array}{c}0 \\
(0 ; 0)\end{array}$ \\
\hline $12-23$ & 28 & 8 & 0,76 & $\begin{array}{c}0,91 \\
0,86 ; 0,95\end{array}$ & $\begin{array}{c}0,03 \\
(-0,75 ; 0,81)\end{array}$ & $\begin{array}{c}0,78 \\
0,60 ; 0,89\end{array}$ & $\begin{array}{c}-0,03 \\
(-0,39 ; 0,33)\end{array}$ \\
\hline $24-35$ & 34 & 7 & 0,40 & $\begin{array}{c}0,72 \\
0,55 ; 0,82\end{array}$ & $\begin{array}{c}-0,21 \\
(-1,3 ; 0,90)\end{array}$ & $\begin{array}{c}0,48 \\
0,19 ; 0,70\end{array}$ & $\begin{array}{c}-0,06 \\
(-0,52 ; 0,41)\end{array}$ \\
\hline $36-47$ & 29 & 4 & 0,23 & $\begin{array}{c}0,60 \\
0,45 ; 0,72\end{array}$ & $\begin{array}{c}-0,04 \\
(-0,95 ; 0,87)\end{array}$ & $\begin{array}{c}0,53 \\
0,22 ; 0,75\end{array}$ & $\begin{array}{c}0,14 \\
(-0,56 ; 0,84)\end{array}$ \\
\hline $48-59$ & 63 & 9 & 0,60 & $\begin{array}{c}0,69 \\
0,54 ; 0,80\end{array}$ & $\begin{array}{c}-0,11 \\
(-1,43 ; 1,21)\end{array}$ & $\begin{array}{c}0,67 \\
0,51 ; 0,79\end{array}$ & $\begin{array}{c}0,05 \\
(-0,6 ; 0,7)\end{array}$ \\
\hline Total & 224 & 38 & --- & $\begin{array}{c}0,75 \\
0,69 ; 0,80\end{array}$ & $\begin{array}{c}0,05 \\
(-0,62 ; 0,71)\end{array}$ & $\begin{array}{c}0,70 \\
0,63 ; 0,76\end{array}$ & $\begin{array}{c}-0,03 \\
(-0,43 ; 0,48)\end{array}$ \\
\hline
\end{tabular}


tre moderados $(0,60)$ para el grupo de 36 a 47 meses y casi perfecta $(0,92)$ para el grupo de 2 a 6 meses. El análisis de Bland y Altman mostró promedios de las diferencias cercanos a cero con límites estrechos para la mayoría de los grupos etarios. La reproducibilidad fue substancial para la detección de rezagos y retrasos.

\section{Validez de constructo convergente del DIEH vs la EAD}

Se encontró una correlación casi perfecta $(\rho=0,96)$ entre los puntajes totales de los dos instrumentos (figura 2); sin embargo, los puntajes condicionados a la edad tuvieron una gran variabilidad, con coeficientes de correlación entre 0,20 y 0,78 ; los más bajos se registraron para los grupos de 25 a 36 meses y de 37 a 48 meses; se encontraron correlaciones moderadas para los grupos etarios de 7 a 12 meses, 13 a 24 meses y 49 a 60 meses y alta para el grupo entre 0 y 6 meses (tabla 3 ). Discusión

Este es el primer estudio en Latinoamérica que realiza una adaptación cultural y, además, evalúa las propiedades psicométricas del DIEH, generando una oportunidad para mostrar el potencial de un cuestionario para estimar la situación del desarrollo infantil en niños entre 1 y 5 años mediante encuestas nacionales en condiciones de tamizaje, a bajo costo y en forma rápida y sencilla.

A partir de la aplicación del DIEH se encontró una prevalencia de retrasos de $11,2 \%$ y de rezagos de 38,8\%, esta última fue superior a la reportada en la II Encuesta Nacional de Salud y Calidad de Vida en hogares (ENCAVI) en Chile en $2006^{11}$, diferencias que pueden deberse a la base poblacional y representatividad nacional de la ENCAVI, a diferencia de nuestro estudio. Por el contrario, se encontraron prevalencias semejantes con el cuestionario de Evaluación del Desarrollo Infantil (EDI) en $\mathrm{México}^{27}$, diseñado para ser aplicado en condiciones de tamizaje con ítems similares.

En cuanto a las diferencias en las prevalencias de retrasos detectadas con la DIEH $(11,2 \%)$ comparadas con las alertas de la EAD (18,7\%), pueden obedecer en primer lugar a las condiciones de aplicación de ambos cuestionarios, es decir tamizaje y diagnóstico, respectivamente; en segundo lugar, quien responde la encuesta corresponde a los padres o cuidadores vs., un profesional de la salud entrenado, quien realiza la valoración directa, también respectivamente. En tercer lugar, la estructura de cada cuestionario, pues la EAD cuenta con igual número de ítems por área de desarrollo y grupo etario, por el contrario, la DIEH presenta un número variable de ítems. Estas diferencias pueden conducir a puntajes, clasificaciones y prevalencias diferentes.

Es posible que el estrato socioeconómico explique en parte las prevalencias de rezagos y retrasos encon-

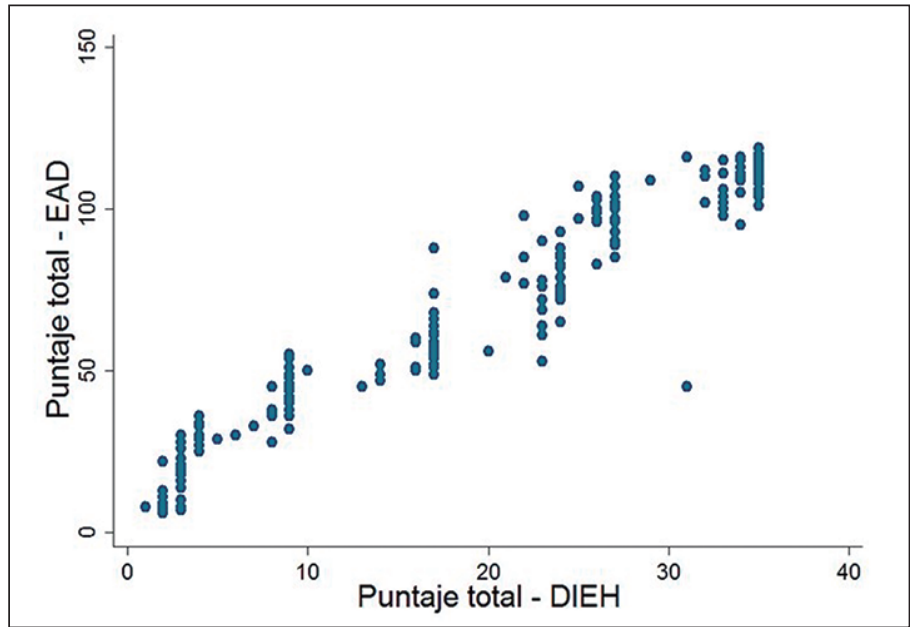

Figura 2. Correlación entre el puntaje total general de la Escala Abreviada del Desarrollo y el Cuestionario de Tamizaje de Desarrollo Infantil para Encuestas en Hogares (rho: 0,96 $p<0,001$ ).

Tabla 3. Coeficientes de correlación de Spearman ( $\rho$ ) entre el puntaje total y condicionado a la edad de la Escala Abreviada del Desarrollo (EAD) y del Cuestionario de Tamizaje de Desarrollo Infantil para Encuestas en Hogares (DIEH)

\begin{tabular}{cccc}
\hline $\begin{array}{c}\text { Grupo etario } \\
\text { (meses) }\end{array}$ & $n$ & $\begin{array}{c}\text { Coeficiente de correlación } \\
(\rho)\end{array}$ & $p$ \\
\hline $0-6$ & 31 & 0,78 & $<0,001$ \\
$7-12$ & 39 & 0,69 & $<0,001$ \\
$13-24$ & 28 & 0,31 & 0,110 \\
$25-36$ & 30 & 0,24 & 0,196 \\
$37-48$ & 29 & 0,10 & 0,587 \\
$49-60$ & 62 & 0,36 & 0,004 \\
Total & 219 & 0,96 & $<0,001$ \\
\hline
\end{tabular}

tradas, ya que el 63\% de la muestra correspondió a los estratos más bajos ( 1 y 2), factor que, en estudios previos, se ha encontrado asociado positivamente con alteraciones en el desarrollo infantil, principalmente con las habilidades de lenguaje $\mathrm{e}^{28,29}$.

En relación con la consistencia interna, pocos estudios registran datos de cuestionarios de tamizaje dirigidos a los padres. Uno de los más estudiados es el Ages and Stages Questionnaire (ASQ) con coeficientes $\alpha$ de Cronbach entre 0,49 y 0,87 en varias poblaciones ${ }^{30-33}$. Así mismo, el Child Development Inventory (CDI) diseñado para niños entre 15 y 72 meses, que contiene de 3 a 8 ítems por grupo etario y toma de 30 a 50 minutos su aplicación, mostró $\alpha$ entre 0,8 y $0,9^{34}$.

Es importante señalar que el DIEH no contaba con una evaluación previa de su consistencia interna. No obstante, nuestros hallazgos son similares al ASQ y el 
CDI, aunque varían entre los grupos etarios, con $\alpha$ entre 0,23 en el grupo de 36 a 47 meses, hasta 0,76 en el grupo de 12-23 meses; este amplio rango de coeficientes puede obedecer a varios factores. En primer lugar, el $\alpha$ de Cronbach se afecta por el número de ítems evaluados, que varía desde 4 ítems con $\alpha$ : 0,23 hasta 9 ítems con $\alpha$ : 0,60; en segundo lugar, se afecta por las características de la población en la que se evalúe y en tercer lugar, lo que se determina es la correlación interna de los ítems en cada dominio o subgrupo evaluado ${ }^{35}$.

La comparación de nuestros hallazgos de reproducibilidad con otros cuestionarios de tamizaje para padres es limitada. El CCI para el puntaje total $(0,75)$, fue inferior al ASQ con CCI entre 0,75 y $0,94^{36,37}$. Armijo y cols. ${ }^{33}$, reportaron coeficientes de correlación de Pearson entre 0,73 y 0,94 en niños de 8 y 18 meses para el ASQ, aunque cabe señalar, que este coeficiente evalúa asociación y no reproducibilidad, al igual que el porcentaje de acuerdo, que en nuestro trabajo osciló entre $62,1 \%$ y $76,9 \%$, comparadas con el ASQ entre $92 \%$ y $94 \%{ }^{30,37}$; nuevamente cabe aclarar, que el porcentaje de acuerdo tampoco evalúa reproducibilidad, dado que no corrige aquella encontrada por azar, como la que se obtiene con la prueba Kappa de Cohen. De otro lado, el propósito del DIEH es contar con información poblacional sobre la condición de desarrollo infantil mediante un tamizaje, mientras que el ASQ es aplicado con mayor detalle a los padres y tiene como propósito su participación activa en el proceso de crecimiento y desarrollo de su hijo.

En cuanto a la validez de constructo convergente entre ambos cuestionarios, fue casi perfecta $(\rho=0,96)$, sin embargo, al estratificar según el grupo etario se observó una disminución progresiva con el aumento de la edad, con excepción del grupo de 49 a 60 meses. La comparación de estos hallazgos con otros estudios no fue posible, pues no se encontraron estudios en la literatura revisada; no obstante, es posible que la baja correlación observada en algunos grupos etarios se deba a la distribución diferencial de ítems por área de desarrollo según el tramo de edad, puesto que la EAD cuenta con tres preguntas por área y grupo etario, mientras que la DIEH tiene un número variable de ítems por grupo etario y no discrimina el área de evaluación.

Entre las limitaciones del estudio cabe señalar el limitado tamaño de muestra por grupo etario, que disminuye el nivel de precisión de los estimados; de otro lado, la muestra no cuenta con representatividad poblacional, dada la vinculación de los participantes con los Centros del Instituto Colombiano de Bienestar Familiar de la ciudad, lo que dejaría por fuera población infantil con características sociodemográficas diferentes y generaría posiblemente, prevalencias de rezagos, retrasos y alertas más bajas.
No obstante, es importante señalar que, en general, la reproducibilidad encontrada para el DIEH muestra hallazgos prometedores para su aplicación en encuestas nacionales, datos que son complementarios con los hallazgos de la validez de constructo convergente analizada.

\section{Conclusión}

Este trabajo aporta información relevante sobre la confiabilidad y validez del DIEH y, plantea que la adaptación cultural de este cuestionario para Colombia y otros países de Latinoamérica y el Caribe, podría ser una herramienta útil para el tamizaje de alteraciones del desarrollo infantil en encuestas nacionales; sin embargo, se requieren estudios adicionales para recomendar su uso ampliado. Finalmente, es importante destacar la necesidad de disponer de un sistema formal para la vigilancia del desarrollo infantil a nivel estatal, con el fin de favorecer la toma de decisiones en política pública, destinada a garantizar el diagnóstico y atención oportuna que favorezcan la inclusión y participación de esta población en condiciones de equidad ${ }^{38}$.

\section{Responsabilidades Éticas}

Protección de personas y animales: Los autores declaran que los procedimientos seguidos se conformaron a las normas éticas del comité de experimentación humana responsable y de acuerdo con la Asociación Médica Mundial y la Declaración de Helsinki.

Confidencialidad de los datos: Los autores declaran que han seguido los protocolos de su centro de trabajo sobre la publicación de datos de pacientes.

Derecho a la privacidad y consentimiento informado: Los autores han obtenido el consentimiento informado de los pacientes y/o sujetos referidos en el artículo. Este documento obra en poder del autor de correspondencia.

\section{Financiamiento}

El proyecto fue financiado por la Vicerrectoría de Investigación y Extensión de la Universidad Industrial de Santander, código 1814. Bucaramanga, Colombia.

\section{Conflicto de intereses}

Los autores declaran no tener conflicto de intereses. 


\section{Referencias}

1. Tecklin J. Pediatric physical therapy. $5^{\mathrm{a}}$ ed. Philadelphia: Lippincott Williams \& Wilkins;2015.

2. Brofenbrenner U. The ecology of human development. Cambridge: Harvard University Press; 1979.

3. Bedregal P, Scharager J, Breinbauer C, Solari J, Molina H. El desarrollo de un modelo para la evaluación de rezagos del desarrollo infantil de Chile. Rev Med Chile 2007;135:403-5.

4. Banco Interamericano de Desarrollo. Early childhood stimulation interventions in early childhood stimulation interventions in developing countries: A comprehensive literature review [Internet]. 2010. [Consultado: 15 de enero de 2019]. Disponible en: https:// www.econstor.eu/handle/10419/89041.

5. Heckman JJ. Invest in the very young. Policy Stud [Internet]. 2000. Disponible en: https://www.ounceofprevention.org/ news/pdfs/HeckmanInvestInVeryYoung. pdf.

6. Black MM, Walker SP, Fernald LCH, et al. Early childhood development coming of age: Science through the life course. Lancet. 2017;389(10064):77-90.

7. Vericat A, Orden AB. Herramientas de screening del desarrollo psicomotor en Latinoamérica. Rev Chil Pediatr. 2010;81(5):391-401.

8. Álvarez MJ, Soria J, Galbe J. Importancia de la vigilancia del desarrollo psicomotor por el pediatra de Atención Primaria: Revisión del tema y experiencia de seguimiento en una consulta en Navarra. Pediatr Aten Primaria. 2009;11(41):65-87.

9. Lejarraga $\mathrm{H}$, Menéndez A, Menzano E, et al. PRUNAPE: pesquisa de trastornos del desarrollo psicomotor en el primer nivel de atención. Arch Argent Pediatr. 2008;106(2):119-25.

10. Tirado-Callejas KB, Arvizu-Mejí LM, Martínez-Pacheco MA, et al. Prevalencia de alteraciones en el desarrollo psicomotor para niños de 1 mes a 5 años valorados con la prueba EDI en un centro de salud en México en el periodo febrero a noviembre de 2015. Eur Sci J. 2017;13(3):223-34.

11. Gobierno de Chile. Ministerio de Salud. II Encuesta de calidad de vida y salud. Informe de resultados [Internet]. 2006. [Consultado: 15 de enero de 2019]. Disponible en: http://www.crececontigo. gob.cl/wp-content/uploads/2015/11/ ENCAVI-2006.pdf

12. American Academy of Pediatrics, Council on Children with Disabilities, Section on
Developmental Behavioral Pediatrics, Bright Futures Steering Committee, Medical Home Initiatives For Children With Special Needs Project Advisory Committee. Identifying infants and young children with developmental disorders in the medical home: an algorithm for developmental surveillance and screening. Pediatrics, 2006;118(1):405-20.

13. Lejarraga H. Desarrollo del niño en contexto. Buenos Aires: Paidós SAICF; 2004.

14. Frankenburg W. Developmental surveillance and screening of infants and young children. Pediatrics. 2002;109:144-5.

15. Malerba K. Assessment and testing of infant and child development. En: Tecklin J. Pediatric Physical Therapy. $5^{\mathrm{a}}$ ed. Baltimore; 2015.

16. Bedregal P. Instrumentos de medición del desarrollo en Chile. Rev Chil pediatría. 2008;79(1):32-6.

17. Moreno S, Ganados C, Rodríguez N, Gómez C. Escala Abreviada de Desarrollo. Pontificia Universidad Javeriana [Internet]. 2016. [Consultado: $15 \mathrm{de}$ enero de 2019]. Disponible en: https:// www.minsalud.gov.co/sites/rid/Lists/ BibliotecaDigital/RIDE/VS/PP/ENT/ Escala-abreviada-de-desarrollo-3.pdf

18. Hormiga CM, Camargo DM, Orozco LC. Reproducibilidad y validez convergente de la Escala Abreviada del Desarrollo y una traducción al español del instrumento Neurosensory Motor Development Assessment. Biomédica. 2008;28(3):32746.

19. Romo B, Liendo S, Vargas G, Rizzoli A, Buenrostro G. Pruebas de tamizaje de neurodesarrollo global para niños menores de 5 años de edad validadas en Estados Unidos y Latinoamérica: Revisión sistemática y análisis comparativo. Bol Med Hosp Infant Mex. 2012;69(6):45062.

20. Bedregal $\mathrm{P}$, Hernández V, Yeomans $\mathrm{H}$, Molina H. Validez concurrente de un instrumento simple de evaluación del desarrollo infantil temprano para encuestas de hogares. Rev Med Chil. 2013;141:409-10.

21. Ministerio de Salud de Colombia. Régimen contributivo [Internet] 2018. [Consultado: enero 15 de 2019]. Disponible en: https://www.minsalud.gov. co/proteccionsocial/Regimencontributivo/ Paginas/regimen-contributivo.aspx

22. Ministerio de Salud de Colombia. Régimen subsidiado [Internet] 2018. [Consultado: 15 de enero de 2019]. Disponible en: https://www. minsalud.gov.co/salud/Paginas/
R\%C3\%A9gimenSubsidiado.aspx

23. Ministerio de Salud de Colombia. Norma técnica para la detección temprana de las alteraciones del crecimiento y desarrollo en el menor de 10 años [Internet]. 2015. [Consultado: 15 de enero de 2019]. Disponible en: https://www.minsalud. gov.co/sites/rid/Lists/BibliotecaDigital/ RIDE/VS/PP/6Deteccion\%20 alteraciones\%20del\%20crecimiento.pdf

24. Ministerio de Salud de Colombia. Resolución 412 [Internet]. 2000. [Consultado: 15 de enero de 2019]. Disponible en: https://docs. supersalud.gov.co/PortalWeb/Juridica/ OtraNormativa/R0412000.pdf

25. Landis JR, Koch GG. The measurement of observer agreement for categorical data. Biometrics. 1977;33(1):159-74.

26. Bland JM, Altman DG. Statistical methods for assessing agreement between two methods of clinical measurement. Lancet. 1986;1(8476):307-10.

27. Rizzoli A, Schnaas L, Liendo S, et al. Validación de un instrumento para la detección oportuna de problemas de desarrollo en menores de 5 años en México. Bol Med Hosp Infant Mex. 2013;70(3):195-208.

28. Bradley RH, Corwyn RF. Socioeconomic status and child development. Annu Rev Psychol. 2002;53(1):371-99.

29. Letourneau NL, Duffett-Leger L, Levac L, Watson B, Young-Morris C. Socioeconomic status and child development: A meta-analysis. J Emot Behav Disord. 2011;21(3):1-14.

30. Squires J, Bricker D, Potter L. Revision of a parent-completed development screening tool: Ages and Stages Questionnaires. J Pediatr Psychol. 1997;22(3):313-28.

31. Vameghi R, Sajedi F, Kraskian Mojembari A, Habiollahi A, Lornezhad HR, Delavar B. Cross-cultural adaptation, validation and standardization of Ages and Stages Questionnaire (ASQ) in Iranian children. Iran J Public Health. 2013;42(5):522-8.

32. Kerstjens JM, Bos AF, ten Vergert EMJ, de Meer G, Butcher PR, Reijneveld SA. Support for the global feasibility of the Ages and Stages Questionnaire as developmental screener. Early Hum Dev. 2009;85(7):443-7.

33. Armijo I, Schonhaut L, Cordero M. Validation of the Chilean version of the Ages and Stages Questionnaire (ASQ-CL) in Community Health Settings. Early Hum Dev. 2015;91:671-6.

34. Ireton H, Glascoe FP. Assessing children's development using parents' reports. The Child Development Inventory. Clin Pediatr (Phila). 1995;34(5):248-55. 
35. Orozco LC. Medición en Salud. Diagnóstico y evaluación de resultados. Bucaramanga-Colombia: División de publicaciones UIS; 2010.

36. Singh A, Squires J, Yeh C, Heo K, Bian $\mathrm{H}$. Validity and reliability of the developmental assessment screening scale. J Fam Medicine Prim Care. 2016;5(1):124-30.

37. Squires J, Twombly E, Bricker D, Potter L. Ages \& Stages Questionnaires. ASQ-3 User's guide. Third Edition. Baltimore:
Brookes Publishing Company; 2009.

38. Mukherjee S, Aneja S, Krishnamurthy V, Srinivasan R. Incorporating developmental screening and surveillance of young children in office practice. Indian Pediatr. 2014;51(8):627-35. 